tant à cause des nombreuses occupations de ses membres, que par suite de la circonstance que plasieurs des personnes qui en faisaient partie ont quitté Belgrade (entro antres M. G. Simitch, nommé agent politique de Serbie a Sofia).

Le Comité central ne doule pas que la commission, une fois complétée, ne mène rapidement son auvre à boune fiu.

Durant celle période de trois ans, le Comilé central a tenu vingl-huit séances.

Il a été procédé régnlièrement à la vérification de la caisse et à la révision des comptes, ainsi qu'à l'inventaire des dépôts.

\title{
SUISSE
}

\section{La SOCIÉTÉ des SAMARITAINS A BERNE}

Lors de la fondation de la Société suisse de la Croix-Rouge, il fut décidó, entrantres, d'organiser aussi en Suisse les seconrs à domer en cas d'accidents en temps de paix. L'organisation en fut remise a la Société militaire sanitaire suisse, qui forme une section de la Chroix-Ronge. Les premiers essais devaient avoir lieu à Berne. Je fus chargé par la section militaire sanitaire d'organiser ce service et de présenter à la Croix-Ronge suisse son plan d'action.

A l'Assemblée générale le Lucenre, lo 3 décembre 1883 , je pris la liberté de présęuter ies propositions suivantes, qui furent renvoyées a la Direction pour élnde, avant d'être prises en considération, ce qui eut lien a la séance suivante :

10 De créer à Berne des cours pour Samaritains;

$2^{\circ}$ Les frais seraieut converts par la caisse centrale;

$3^{\circ}$ Il serait fait annuellement une statistique des secours donnés, et la Direction rembourserait, aux sections des Samaritains, leurs debours pour le matériel sanitaire employé;

$4^{\circ}$ La Direction de la Croix-liouge serait priée de faire son possible pour organiser le service sanitaire sur les lignes de chemins de fer.

En exécution du $z$ 1, une assemblée publique eut lieu à la Langgasse, a Berne, et, a la suite d'une conférence sur le but des cours pour Samaritains, 25 hommes se présentèrent pour suivre le premier cours. 
L'organisation de ces cours comprenait :

Une partie théorique, donnée par M. le Dr Bob. Vogl;

- pratique, donnée par le soussigné.

La partie théorique comprenail: l'anatomie (restreinte), le traitement des plaies, les soins urgents à douner en cas de brủlure, d'empoisonnement, etc., etc, ; en un mot, ce qu'il est nécessatire de savoir pour porter secours an premier moment.

La partie pratique concernait: les pansements d'urgence et d'ordonnance, le transport, etc., etc.

Chaque cours dura de 7 à 8 semaines, à raison de deux séances de deux heures par semaine, dont l'une consacrée à la théorie et l'antre à la pratique.

A la fin de chaque conrs, les Samaritains passèrent un examen public. M. le colonel Dr Ziegler, médecin en chef, a bien voulu les examiner, et, sur sa proposition, tous les aspirants ont été diplomés. Nous avons donné 4 cours et instruit environ 90 hommes, qui șont répartis dans les dilférents quartiers de la ville et des environs.

la Direction de la Croix-Rouge a payé 100 francs pour le premier et le second cours. Pour les deux antres, une partie des frais a été couverte par les particuliers des différents quartiers, et le reste par la Direction de l'intérieur du Canton et par la commune de Berne.

Nous avous actuellernent cinq postes sauitaires:

$1^{\circ}$ Langgasse; $2^{\circ}$ Lorraine; $3^{\circ}$ Matle; $4^{\circ}$ Mattenhof; $5^{\circ}$ Linde.

Ces postes sont tenus gratuitement par des Samaritains.

Les postes sont désignés par une affiche, avec l'inscription: Samariter-Posten - Mat rial Depot, et chacun d'eux a à sa disposition une cassette sanitaire, renfermant: handes, triangles, ouate, charpie, jute, enfin tont le nécessaire pour donner les premiers secours; de plus: un brancard à deux rones, un matelas, un traversin et une couverture, pour le transport des blessés. Chaque Samaritain est en outre pourvu d'une cartouche à pansement.

Les Samaritains out fondé une socièté, qui a pour but de répéter mensuellement l'iustruction reçe. Colte société se divise en quatre quartiers ou sections, dont chacune est dirigée et instruite par un sous-officier sanilaire pour les exercices pratiques. Un comité central règle les questions administratives et ponrvoit à toutes les dépenses nécessaires. Les statuts sont en préparation. Les nom- 
brenx frais pour le materiel sont converts par les dons et colisations des membres actifs el passifs.

La Croix-Rouge suisse nous ayant retiré son concours financier i la fin du troisieme cours, nous avons adressé an jublic de notre ville un appel qui a produit la leelle somme de 500 frames et plus, el rui nous a jermis d'organiser nos postes comvablenent. La Sociélé possede en outre une bibliothègue, qui compte actuellentent $16($ ) volnmes (tons dons des anis de notre duvre), el jons avons l'espoir de la voir' s'accroître bientòt.

Les secours domnés en cas d'aucidents dépassent le cliffre de 80, II y a eu plusieurs cas graves, lels que fraclures simples el compliquées, chules, efc., qui ont donné à nos Samarilains l'occasion de montrer leurs comnaissances. Nolre organisalion ent très goultée de nos concitoyens el actuellement on s'en passerail dillicilement. Les subventions regnes, mône des medecins de Berne, prouvent qu'ils sont persuadés que nons n'enjpićtons pais sur leur domaine, mais que nous nous en lenons a notre règlement qui dit que : "Tous antres secours que les premiers, en ras d'accident, sont inlerdits anx membres de la Société."

Nous avons, conme signe distinclif, me cocarde, qui nons a été remise el qui est recomme par la commission de santé el de police a Berne. En cas d'incendie, nous sommes a la disposition des médecins du corps des sapeurs-pompiers.

Pour terminer, je note encore que l'instruction domnée dans les cours a été gratuile, et que tous nos services, ainsi que le matériel sanitaire employé pour les pansemenls, sont fournis gratuitement.

Notre devise est:

" Citre ulite à son prochain en cas d'accident, el fréparer, en temps de paix, une organisation qui pourrail ctre appelée à rendre de grands services en temps de guerre.

Limest Mockly

sergent-major infirmier, président de la Société militaire sunitaire at de la Société des Samaritains, à Bene. 